\title{
ON A CONJECTURE OF GACKSTATTER AND LAINE
}

\author{
By Mitsuru OzAwa
}

Dedicated to Professor Shigeru Ishihara on his 60th birthday

$\S 1$. Introduction. In their joint paper [2] Gackstatter and Laine offered the following conjecture: Let $a_{\imath}(z), \imath=0,1, \cdots, n-k$, be meromorphic in $|z|<\infty$. Then

$$
w^{\prime n}=\sum_{i=0}^{n-k} a_{i}(z) w^{\imath}
$$

does not admit any admissible solution, where $a_{n-k}(z) \not \equiv 0$ and $k$ is an integer satisfying $1 \leqq k \leqq n-1$. Here the admissible solution means a meromorphic solution of the given equation satisfying

$$
T\left(r, a_{\imath}(z)\right)=o(T(r, w))
$$

for all $\imath$ except for at most a set $E$ of $r$ of finite measure. In what follows this is simply denoted by $T\left(r, a_{\imath}\right)=S(r, w)$.

The above conjecture has a close connection with an unsolved problem due to Hayman ([4] Problem 1.21). Indeed, if the conjecture is true, then the simplest case $w^{\prime}=a_{0}(z)$ implies that $T\left(r, w^{\prime}\right)=S(r, w)$ does not hold. However this is still unsolved, so far as the present author knows. Another simple case is $w^{\prime n}=a(w+\alpha)^{n-k}$ with a constant $\alpha$ and an integer $k(1 \leqq k \leqq n-1)$. In this case

$$
w+\alpha=\left(\frac{n}{k}\right)^{n / k}\left(\int^{z} a^{1 / n} d z+C\right)^{n / k} .
$$

Still we cannot decide whether $T\left(r, w^{\prime}\right)=S(r, w)$ or not.

In this paper we shall give a method to attack the above conjecture and prove the following

TheOREM. Let $a_{j}(z), j=0,1,2,3$, be meromorphic in $|z|<\infty$. Then

$$
w^{\prime n}=a_{3} w^{3}+a_{2} w^{2}+a_{1} w+a_{0}, \quad n \geqq 4, \quad a_{3} \neq 0
$$

does not admit any admissible solution with an exception of the following equation

$$
w^{\prime n}=a_{3}(w+\alpha)^{3}
$$

with a constant $\alpha$.

Received March 24, 1982 
$\S 2$. Proof of Theorem. As Gackstatter and Laine remarked in [2] every meromorphic solution $w$ does not have any pole other than those of coefficients and the multiplicities of poles of $w$ are less than those of coefficients. Hence

$$
N(r, w)=S(r, w), \quad N\left(r, w^{\prime}\right)=S(r, w)
$$

for every admissible solution $w$. Further $n m\left(r, w^{\prime}\right)=3 m(r, w)+S(r, w)$. Hence $S(r, w)=S\left(r, w^{\prime}\right)$. We may assume that $n=4$, since the general case $n$ can be treated quite similarly. Thus we start from

$$
w^{\prime 4}=a_{3} w^{3}+a_{2} w^{2}+a_{1} w+a_{0} .
$$

By differentiation of this equation we have

$$
4 w^{\prime 3} w^{\prime \prime}=a_{3}{ }^{\prime} w^{3}+\left(a_{2}{ }^{\prime}+3 a_{3} w^{\prime}\right) w^{2}+\left(a_{1}{ }^{\prime}+2 a_{2} w^{\prime}\right) w+a_{0}{ }^{\prime}+a_{1} w^{\prime} .
$$

From the above two equations we eliminate $w$. Then we have

$$
\left(\alpha_{0}+\alpha_{1} w^{\prime}+\alpha_{2} w^{\prime 2}+A\right)\left(\delta_{0}+\delta_{1} w^{\prime}+\delta_{2} w^{\prime 2}+D\right)=\left(\beta_{0}+\beta_{1} w^{\prime}+\beta_{2} w^{\prime 2}+B\right)^{2},
$$

where

with

$$
\begin{aligned}
\alpha_{0}= & -a_{1} X^{2}+a_{2} X Y+a_{3} X Z-a_{3} Y^{2}, \\
\alpha_{1}= & \left(2 a_{2}{ }^{2} a_{3}-5 a_{1} a_{3}{ }^{2}\right) X-a_{2} a_{3}{ }^{2} Y+3 a_{3}{ }^{3} Z, \\
\alpha_{2}= & 2 a_{2}{ }^{2} a_{3}{ }^{3}-6 a_{1} a_{3}{ }^{4}, \\
\beta_{0}= & a_{0} X^{2}-a_{2} X Z+a_{3} Y Z, \\
\beta_{1}= & \left(6 a_{0} a_{3}{ }^{2}-a_{1} a_{2} a_{3}\right) X+a_{1} a_{3}{ }^{2} Y-a_{2} a_{3}{ }^{2} Z, \\
\beta_{2}= & 9 a_{0} a_{3}{ }^{4}-a_{1} a_{2} a_{3}{ }^{3}, \\
\delta_{0}= & a_{1} X Z-a_{0} X Y-a_{3} Z^{2}, \\
\delta_{1}= & \left(a_{1}{ }^{2} a_{3}-2 a_{0} a_{2} a_{3}\right) X-3 a_{0} a_{3}{ }^{2} Y+a_{1} a_{3}{ }^{2} Z, \\
\delta_{2}= & 2 a_{1}{ }^{2} a_{3}{ }^{3}-6 a_{0} a_{2} a_{3}{ }^{3}, \\
A= & a_{3}\left(X+3 a_{3}{ }^{2} w^{\prime}\right)\left(a_{3}{ }^{\prime} w^{\prime 4}-4 a_{3} w^{\prime 3} w^{\prime \prime}\right), \\
B= & -\left(X+3 a_{3}{ }^{2} w^{\prime}\right)^{2} w^{\prime 4}-\left(a_{2} X-a_{3} Y+a_{2} a_{3}{ }^{2} w^{\prime}\right)\left(a_{3}{ }^{\prime} w^{\prime 4}-4 a_{3} w^{\prime 3} w^{\prime \prime}\right), \\
D= & -a_{3}\left(a_{3}{ }^{\prime} w^{\prime 4}-4 a_{3} w^{\prime 3} w^{\prime \prime}\right)^{2}+\left(X+3 a_{3}{ }^{2} w^{\prime}\right)\left(Y+2 a_{2} a_{3} w^{\prime}\right) w^{\prime 4} \\
& +\left(a_{1} X-2 a_{3} Z+a_{1} a_{3}{ }^{2} w^{\prime}\right)\left(a_{3}{ }^{\prime} w^{\prime 4}-4 a_{3} w^{\prime 3} w^{\prime \prime}\right)
\end{aligned}
$$

$$
X=a_{3} a_{2}{ }^{\prime}-a_{3}{ }^{\prime} a_{2}, \quad Y=a_{3} a_{1}{ }^{\prime}-a_{3}{ }^{\prime} a_{1}, \quad Z=a_{3} a_{0}{ }^{\prime}-a_{3}{ }^{\prime} a_{0} .
$$

Let $\left\{z_{n}\right\}$ be the set of zeros of $w^{\prime}$ for which all of $a_{\jmath} \neq \infty$. This set may be 
void or scarce so that $N\left(r, 0, w^{\prime}\right)=S\left(r, w^{\prime}\right)$. If this is not, then from (A)

$$
\alpha_{0} \delta_{0}=\beta_{0}{ }^{2}
$$

at $\left\{z_{n}\right\}$. If (1) is not an identity, then

$$
N\left(r, 0, w^{\prime}\right) \leqq N\left(r, 0, \alpha_{0} \delta_{0}-\beta_{0}{ }^{2}\right)+S\left(r, w^{\prime}\right)=S\left(r, w^{\prime}\right) .
$$

If (1) is an identity, then from (A)

$$
\alpha_{0} \delta_{1}+\alpha_{1} \delta_{0}=2 \beta_{0} \beta_{1}
$$

at $\left\{z_{n}\right\}$. If (2) is not an identity, then $N\left(r, 0, w^{\prime}\right)=S\left(r, w^{\prime}\right)$. If (2) is an identity, then from $(\mathrm{A})$

$$
\alpha_{0} \delta_{2}+\alpha_{1} \delta_{1}+\alpha_{2} \delta_{0}=2 \beta_{0} \beta_{2}+\beta_{1}{ }^{2}
$$

at $\left\{z_{n}\right\}$. If (3) is not an identity, then $N\left(r, 0, w^{\prime}\right)=S\left(r, w^{\prime}\right)$.

Assume firstly that (1), (2) and (3) are identities. (1) gives either $X=0$ or

$$
\begin{gathered}
a_{0}{ }^{2} X^{3}-a_{0} a_{1} X^{2} Y+\left(a_{1}{ }^{2}-2 a_{0} a_{2}\right) X^{2} Z+a_{0} a_{2} X Y^{2}+\left(3 a_{0} a_{3}-a_{1} a_{2}\right) X Y Z \\
+\left(a_{2}{ }^{2}-2 a_{1} a_{3}\right) X Z^{2}-a_{0} a_{3} Y^{3}+a_{1} a_{3} Y^{2} Z-a_{2} a_{3} Y Z^{2}+a_{3}{ }^{2} Z^{3}=0 .
\end{gathered}
$$

(2) gives

$$
\begin{aligned}
& \left(-a_{1}{ }^{3}+4 a_{0} a_{1} a_{2}-12 a_{0}{ }^{2} a_{3}\right) X^{3}+\left(a_{1}{ }^{2} a_{2}-4 a_{0} a_{2}{ }^{2}+6 a_{0} a_{1} a_{3}\right) X^{2} Y \\
& \quad+\left(12 a_{0} a_{2} a_{3}-5 a_{1}{ }^{2} a_{3}\right) X^{2} Z-a_{1}{ }^{2} a_{3} X Y^{2}+\left(4 a_{1} a_{2} a_{3}-18 a_{0} a_{3}{ }^{2}\right) X Y Z \\
& \quad+\left(9 a_{1} a_{3}{ }^{2}-4 a_{2}{ }^{2} a_{3}\right) X Z^{2}+3 a_{0} a_{3}{ }^{2} Y^{3}-3 a_{1} a_{3}{ }^{2} Y^{2} Z+3 a_{2} a_{3}{ }^{2} Y Z^{2}-3 a_{3}{ }^{3} Z^{3}=0
\end{aligned}
$$

(3) is equivalent to

$$
\begin{aligned}
\left(30 a_{0}\right. & \left.a_{1} a_{2} a_{3}-7 a_{1}{ }^{3} a_{3}-4 a_{0} a_{2}{ }^{3}+a_{1}{ }^{2} a_{2}{ }^{2}-54 a_{0}{ }^{2} a_{3}{ }^{2}\right) X^{2} \\
& +\left(3 a_{1}{ }^{2} a_{2} a_{3}-12 a_{0} a_{2}{ }^{2} a_{3}+9 a_{0} a_{1} a_{3}{ }^{2}\right) X Y+\left(18 a_{0} a_{2} a_{3}{ }^{2}-6 a_{1}{ }^{2} a_{3}{ }^{2}\right) X Z \\
& +\left(9 a_{0} a_{2} a_{3}{ }^{2}-3 a_{1}{ }^{2} a_{3}{ }^{2}\right) Y^{2}+\left(3 a_{1} a_{2} a_{3}{ }^{2}-27 a_{0} a_{3}{ }^{3}\right) Y Z \\
& +\left(9 a_{1} a_{3}{ }^{3}-3 a_{2}{ }^{2} a_{3}{ }^{2}\right) Z^{2}=0 .
\end{aligned}
$$

The case $X=0$ will be considered later. Let us make the following expression $3 a_{3}(4)+(5)$. Then we have either $X=0$ or

$$
\begin{gathered}
\left(4 a_{0} a_{1} a_{2}-a_{1}{ }^{3}-9 a_{0}{ }^{2} a_{3}\right) X^{2}+\left(a_{1}{ }^{2} a_{2}-4 a_{0} a_{2}{ }^{2}+3 a_{0} a_{1} a_{3}\right) X Y \\
\quad+\left(6 a_{0} a_{2} a_{3}-2 a_{1}{ }^{2} a_{3}\right) X Z+\left(3 a_{0} a_{2} a_{3}-a_{1}{ }^{2} a_{3}\right) Y^{2} \\
+\left(a_{1} a_{2} a_{3}-9 a_{0} a_{3}{ }^{2}\right) Y Z+\left(3 a_{1} a_{3}{ }^{2}-a_{2}{ }^{2} a_{3}\right) Z^{2}=0 .
\end{gathered}
$$

Making the expression (6) $-3 a_{3}(7)$, we have either $X=0$ or

$$
\mathscr{D} \equiv 18 a_{0} a_{1} a_{2} a_{3}-4 a_{1}{ }^{3} a_{3}-4 a_{0} a_{2}{ }^{3}+{a_{1}}^{2} a_{2}{ }^{2}-27 a_{0}{ }^{2} a_{3}{ }^{2}=0,
$$


which is equivalent to

$$
\left(27 a_{0}^{2} a_{3}+2 a_{1}^{3}-9 a_{0} a_{1} a_{2}\right)^{2}=4\left(a_{1}^{2}-3 a_{0} a_{2}\right)^{3} .
$$

Further $\mathscr{D}$ is the discriminant of $a_{0} x^{3}+a_{1} x^{2}+a_{2} x+a_{3}$. Hence $\mathscr{D} \equiv 0$ implies that $a_{0} x^{3}+a_{1} x^{2}+a_{2} x+a_{3}=a_{0}\left(x-\varepsilon_{1}\right)^{2}\left(x+\varepsilon_{2}\right)$ with

$$
3 a_{0} \varepsilon_{1}=-a_{1} \pm \sqrt{y}, \quad 3 a_{0} \varepsilon_{2}=a_{1} \pm 2 \sqrt{ } y, \quad y=a_{1}{ }^{2}-3 a_{0} a_{2} .
$$

Since $4 y^{3}$ is equal to $\left(27 a_{0}{ }^{2} a_{3}+2 a_{1}{ }^{3}-9 a_{0} a_{1} a_{2}\right)^{2}, \sqrt{ } y$ is one-valued meromorphic in $|z|<\infty$. Hence so are $\varepsilon_{1}, \varepsilon_{2}$. Returning back to the original eqation, we have

$$
\begin{aligned}
w^{\prime 4} & =a_{0}\left(1-\varepsilon_{1} w\right)^{2}\left(1+\varepsilon_{2} w\right) \\
& =a_{3}\left(w--1 / \varepsilon_{1}\right)^{2}\left(w+1 / \varepsilon_{2}\right) .
\end{aligned}
$$

Suppose $y \neq 0$, that is, $\varepsilon_{2} \neq-\varepsilon_{1}$. Then we have

$$
\Theta\left(1 / \varepsilon_{1}, w\right)+\Theta\left(-1 / \varepsilon_{2}, w\right)+\delta(\infty, w) \geqq 1 / 2+3 / 4+1>2,
$$

which is evidently a contradiction by the Nevanlinna theory. Suppose $y=0$. Then $w^{\prime 4}=a_{3}(w+\alpha)^{3}, \alpha=-1 / \varepsilon_{1}$. In this case by differentiation and by elimination of $w$ successively we have either $w^{\prime}=0$ or

$$
\left(4 a_{3} w^{\prime \prime}-a_{3}{ }^{\prime} w^{\prime}\right)^{3} w^{\prime}=27 a_{3}{ }^{4}\left(w^{\prime}+\alpha^{\prime}\right)^{3} .
$$

If $w^{\prime}=0$, then $w$ is a constant, which may be excluded. Let $\left\{z_{n}\right\}$ be the set of zeros of $w^{\prime}$, for which $a_{3} \neq \infty, \alpha^{\prime} \neq \infty$. Then at $\left\{z_{n}\right\} \quad \alpha^{\prime}=0$. If $\left\{z_{n}\right\}$ is scarce or if $\alpha^{\prime} \neq \equiv$, then $N\left(r, 0, w^{\prime}\right)=S\left(r, w^{\prime}\right)$. Further

$$
27 a_{3}\left(1+\frac{\alpha^{\prime}}{w^{\prime}}\right)^{3} \frac{1}{w^{\prime}}=\left(4 \frac{w^{\prime \prime}}{w^{\prime}}-\frac{a_{3}{ }^{\prime}}{a_{3}}\right)^{3} .
$$

Hence $m\left(r, 1 / w^{\prime}\right)=S\left(r, w^{\prime}\right)$. Thus $T\left(r, w^{\prime}\right)=S\left(r, w^{\prime}\right)$, which is clearly impossible. If $\alpha^{\prime} \equiv 0$, then $\alpha$ is a constant. This reduces to $w^{\prime 4}=a_{3}(w+\alpha)^{3}, \alpha=$ const., which is exceptional.

Next we shall consider the case $X=0$. This means that $a_{2}=c a_{3}$ with a constant $c$. Then the original equation can be reduced to

$$
w^{\prime 4}=a_{3}(w+c / 3)^{3}+A_{1}(w+c / 3)+A_{0} .
$$

Let us put $u=w+c / 3$. Then the above equation takes the following form:

$$
u^{\prime 4}=a_{3} u^{3}+A_{1} u+A_{0} .
$$

Thus we may consider the original equation with $a_{2}=0$. In this case (4) and (6) reduce to

$$
\begin{aligned}
& -a_{0} Y^{3}+a_{1} Y^{2} Z+a_{3} Z^{3}=0, \\
& -a_{1}{ }^{2} Y^{2}-9 a_{0} a_{3} Y Z+3 a_{1} a_{3} Z^{2}=0,
\end{aligned}
$$


respectively. From these two identities

$$
Y\left[-3 a_{0} a_{1} Y^{2}+4 a_{1}{ }^{2} Y Z+9 a_{0} a_{3} Z^{2}\right]=0 .
$$

If $Y=0$, then $Z=0$. In this case we have $w^{\prime 4}=a_{3}\left(w^{3}+d w+e\right)$ with constants $d$ and $e$. Now we have three possibilities:

$$
\begin{aligned}
& w^{\prime 4}=a_{3}\left(w-\alpha_{1}\right)\left(w-\alpha_{2}\right)\left(w-\alpha_{3}\right), \quad\left(\alpha_{1}-\alpha_{2}\right)\left(\alpha_{1}-\alpha_{3}\right)\left(\alpha_{2}-\alpha_{3}\right) \neq 0, \\
& w^{\prime 4}=a_{3}\left(w-\alpha_{1}\right)^{2}\left(w-\alpha_{2}\right), \quad \alpha_{1}-\alpha_{2} \neq 0, \\
& w^{\prime 4}=a_{3}\left(w-\alpha_{1}\right)^{3} .
\end{aligned}
$$

The first case is impossible, since

$$
\sum_{\jmath=1}^{3} \Theta\left(\alpha_{\jmath}, w\right)+\delta(\infty, w) \geqq 9 / 4+1>2 .
$$

The second case is impossible too, since

$$
\Theta\left(\alpha_{1}, w\right)+\Theta\left(\alpha_{2}, w\right)+\delta(\infty, w) \geqq 1 / 2+3 / 4+1>2 .
$$

The last case is exceptional. we have

If $-3 a_{0} a_{1} Y^{2}+4 a_{1}^{2} Y Z+9 a_{0} a_{3} Z^{2}=0$, then by $-a_{1}^{2} Y^{2}-9 a_{0} a_{3} Y Z+3 a_{1} a_{3} Z^{2}=0$

$$
\left(27 a_{0}^{2} a_{3}+4 a_{1}^{3}\right) Y Z=0,
$$

when $a_{0} a_{1} \neq 0$. If $Y=0$, then $Z=0$. This case has already been discussed. If $Z=0$, then $Y=0$. This case has been discussed too. If $27 a_{0}{ }^{2} a_{3}+4 a_{1}{ }^{3}=0$, that is, the discriminant of $a_{0} x^{3}+a_{1} x^{2}+a_{3}$ vanishes identically, then

$$
w^{\prime 4}=a_{3}(w-\alpha)^{2}(w-\beta), \quad \alpha=-\frac{3 a_{0}}{2 a_{1}}, \quad \beta=\frac{3 a_{0}}{a_{1}} .
$$

This gives

$$
\Theta(\alpha, w)+\Theta(\beta, w)+\delta(\infty, w) \geqq 1 / 2+3 / 4+1>2,
$$

since $\alpha \equiv \beta$. It is easy to prove that $a_{0} \equiv 0$ (or $a_{1} \equiv 0$ ) leads us to alcontradiction or an exceptional case.

Suppose (1) is not an identity. In this case (A) reduces to

$$
L_{13}(x)=M L_{9}(x)+M^{2} L_{5}(x)+M^{3} L_{1}(x), \quad x=1 / w^{\prime},
$$

where $L_{j}(x)$ is a polynomial of $x$ of degree $j$ and $M$ is $a_{3}{ }^{\prime}-4 a_{3} w^{\prime \prime} / w^{\prime}$. Further the leading coefficient of $L_{13}(x)$ is just $\alpha_{0} \delta_{0}-\beta_{0}{ }^{2} \neq \equiv$. Then $M=a_{3}{ }^{\prime}+4 a_{3} x^{\prime} / x$. By the well-known Clunie lemma $[1] m(r, x)=S\left(r, w^{\prime}\right)$. On the other hand we already remarked that $N\left(r, 0, w^{\prime}\right)=S\left(r, w^{\prime}\right)$. Hence

$$
\begin{aligned}
T\left(r, w^{\prime}\right) & =N\left(r, 0, w^{\prime}\right)+m\left(r, 1 / w^{\prime}\right)+O(1) \\
& =S\left(r, w^{\prime}\right) .
\end{aligned}
$$


This is evidently impossible.

Suppose that (1) is an identity but (2) is not. Then (A) reduces to

$$
L_{12}(x)=M L_{9}(x)+M^{2} L_{5}(x)+M^{3} L_{1}(x), \quad x=1 / w^{\prime} .
$$

The leading coefficient of $L_{12}(x)$ is just $\alpha_{0} \delta_{1}+\alpha_{1} \delta_{0}-2 \beta_{0} \beta_{1} \neq 0$. Hence the same reasoning as in the above case does work. Thus we have a contradiction.

Suppose that (1) and (2) are identities but (3) is not. Then (A) has the following form:

$$
L_{11}(x)=M L_{9}(x)+M^{2} L_{5}(x)+M^{3} L_{1}(x), \quad x=1 / w^{\prime} .
$$

The leading coefficient of $L_{11}(x)$ is $\alpha_{0} \delta_{2}+\alpha_{1} \delta_{1}+\alpha_{2} \delta_{0}-2 \beta_{0} \beta_{2}-\beta_{1}^{2} \neq \equiv$. So we have a contradiction similarly. This completes the proof.

§3. A supplement. We shall give a supplementary result.

Corollary. Let $\rho$ be $\max \left(\rho_{a_{3}}, \rho_{a_{2}}, \rho_{a_{1}}, \rho_{a_{0}}\right)$, where $\rho_{a_{3}}$ is the order of $a_{\jmath}$. Suppose that $\rho$ is finte, then any meromorphic solution of

$$
w^{\prime n}=a_{3} w^{3}+a_{2} w^{2}+a_{1} w+a_{0}, \quad a_{3} \neq 0, \quad n \geqq 4
$$

is of order at most $\rho$.

In order to prove this corollary we still have several things to be assured.

LEMMA 1. Let $A(r)$ and $B(r)$ be convex continuous increasing functions of $\log r$ for $r \geqq r_{0}$. Further let $B(r)$ be of finte order $\lambda$. Suppose that $A(r) \leqq B(r)$ $+O(\log A(r)+\log r)$ holds for $r \notin E$, where $E$ is the set of $r$ of finte linear measure. Then $A(r)$ is of order at most $\lambda$.

The proof of this lemma is very easy. Further this enables us to prove that there are at most two Borel's exceptional functions of the given meromorphic function.

LEMMA 2. Let $w$ be a meromorphic solution of $w^{\prime n}=a w^{3}, n \geqq 4$, where $a$ is meromorphac of finte order $\rho$. Then $w$ is also of order $\rho$.

This is simply a special case of the corresponding fact of algebroid functions.

LEMMA 3. $w^{\prime n}=a\left(w-\alpha_{1}\right)^{2}\left(w-\alpha_{2}\right)$ with different constants $\alpha_{1}, \alpha_{2}$ and $n \geqq 4$ has no meromorphic solution of order greater than that of $a$.

Proof of Lemma 3. Let $w$ be a meromorphic solution of order greater than the order $\rho$ of $a$. Suppose first that $N\left(r, \alpha_{2}, w\right)$ is of order not greater than $\rho$. Let us put $u=w-\alpha_{2}$, then

$$
u^{\prime n}=a\left(u-\beta_{1}\right)^{2} u
$$


This implies that

$$
\begin{aligned}
(1+o(1))(n-1) m(r, 1 / u) & \leqq m(r, 1 / a)+n m\left(r, u^{\prime} / u\right) \\
& \leqq m(r, 1 / a)+O(\log T(r, u)+\log r)
\end{aligned}
$$

for $r \notin E$, where $E$ is the set of linear measure at most 2. Thus for $r \notin E$

$$
\begin{aligned}
(1+o(1))(n-1) T(r, u) \leqq & T(r, 1 / a)+(1+o(1))(n-1) N(r, 0, u) \\
& +O(\log T(r, u)+\log r) .
\end{aligned}
$$

By Lemma $1 T(r, u)$, that is, $w$ is of order at most $\rho$, which is impossible.

Thus we may suppose that $N\left(r, \alpha_{2}, w\right)$ is of order greater than $\rho$. Since the order of $a$ is $\rho$, there are infinitely many zeros of $w-\alpha_{2}$ satisfying $a \neq 0, \infty$. Let $z_{n}$ be one of them. Let $\nu$ be its multiplicity. Then $n(\nu-1)=\nu$. This is evidently impossible.

The same fact holds for $w^{\prime n}=a\left(w-\alpha_{1}\right)\left(w-\alpha_{2}\right)\left(w-\alpha_{3}\right), n \geqq 4$, if $\alpha_{1}, \alpha_{2}, \alpha_{3}$ are different constants.

We still have to consider the case $\mathscr{D} \equiv 0$, which appeared already in the proof of Theorem. In this case we have

where

$$
\begin{aligned}
w^{\prime 4} & =a_{3}\left(w-1 / \varepsilon_{1}\right)^{2}\left(w+1 / \varepsilon_{2}\right) \\
& =a_{3}\left(w-1 / \tilde{\varepsilon}_{1}\right)^{2}\left(w+1 / \tilde{\varepsilon}_{2}\right),
\end{aligned}
$$

$$
\begin{array}{ll}
3 a_{0} \varepsilon_{1}=-a_{1}+t, & 3 a_{0} \varepsilon_{2}=a_{1}+2 t, \\
3 a_{0} \tilde{\varepsilon}_{1}=-a_{1}-t, & 3 a_{0} \tilde{\varepsilon}_{2}=a_{1}-2 t, \\
t^{2}=y=a_{1}{ }^{2}-3 a_{0} a_{2} &
\end{array}
$$

and $t$ is one-valued meromorphic in $|z|<\infty$. Since there exist at most two Borel's exceptional functions of $w$ and $\infty$ is a Borel's exceptional value of $w$, at least three of $N\left(r, 1 / \varepsilon_{1}, w\right), N\left(r,-1 / \varepsilon_{2}, w\right), N\left(r, 1 / \tilde{\varepsilon}_{1}, w\right)$ and $N\left(r,-1 / \tilde{\varepsilon}_{2}, w\right)$ are of order greater than $\rho$. Of course we start from the assumptions that the order of $w$ is greater than $\rho$ and that $y \neq 0$.

For simplicity's sake we assume that they are

$$
N\left(r, 1 / \varepsilon_{1}, w\right), \quad N\left(r,-1 / \varepsilon_{2}, w\right), \quad N\left(r, 1 / \tilde{\varepsilon}_{1}, w\right) .
$$

Let $\left\{z_{n}\right\}$ be the set of roots of $w(z)=1 / \varepsilon_{1}(z)$ and $a_{3}\left(z_{n}\right) \neq 0$, $\infty$. This set is not empty. Then either $w\left(z_{n}\right)=1 / \tilde{\varepsilon}_{1}\left(z_{n}\right)$ or $w\left(z_{n}\right)=-1 / \tilde{\varepsilon}_{2}\left(z_{n}\right)$. If $w\left(z_{n}\right)=1 / \tilde{\varepsilon}_{1}\left(z_{n}\right)$, then $\varepsilon_{1}\left(z_{n}\right)=\tilde{\varepsilon}_{1}\left(z_{n}\right)$ and hence $\varepsilon_{1}(z) \equiv \tilde{\varepsilon}_{1}(z)$, since $\varepsilon_{1}$, $\tilde{\varepsilon}_{1}$ are of order at most $\rho$ but $\left\{z_{n}\right\}$ is of order greater than $\rho$. This gives $y \equiv 0$, which contradicts $y \neq 0$. If $w\left(z_{n}\right)$ $=-1 / \tilde{\varepsilon}_{2}\left(z_{n}\right)$, then $3 t \equiv 2 a_{1}$ similarly. Let $\left\{x_{n}\right\}$ be the set of roots of $w(z)=-1 / \varepsilon_{2}(z)$ and $a_{3}\left(x_{n}\right) \neq 0, \infty$. Then either $w\left(x_{n}\right)=1 / \tilde{\varepsilon}_{1}\left(x_{n}\right)$ or $w\left(x_{n}\right)=-1 / \tilde{\varepsilon}_{2}\left(x_{n}\right)$. If the former case occurs, then $3 t \equiv-2 a_{1}$ similarlg. Hence $t \equiv 0$, that is, $y \equiv 0$, which is impossible. If the latter case occurs, then similarly $y \equiv 0$, which is impossible. 
Therefore $y \equiv 0$. Then

In this case

$$
w^{\prime 4}=a_{3}(w-\alpha)^{3}, \quad \alpha=-3 a_{0} / a_{1} .
$$

$$
\frac{\left(w^{\prime}-\alpha^{\prime}\right)^{3}}{w^{\prime 4}}=-\frac{1}{27 a_{3}}\left(4 \frac{w^{\prime \prime}}{w^{\prime}}-\frac{a_{3}^{\prime}}{a_{3}}\right)^{3}
$$

Hence

$$
(1+o(1)) 4 m\left(r, 1 / w^{\prime}\right) \leqq m\left(r, 1 / a_{3}\right)+O\left(\log T\left(r, w^{\prime}\right)+\log r\right)
$$

for $r \notin E$, where $E$ is the set of $r$ of linear measure at most 2. Let $\left\{z_{n}\right\}$ be the set of zeros of $w^{\prime}$ being of order greater than $\rho$. Then by the above equation $\alpha^{\prime}\left(z_{n}\right)=0$. This gives $\alpha^{\prime}(z) \equiv 0$, that is, $\alpha$ is a constant, if $w^{\prime} \not \equiv 0$. Hence Lemma 2 implies the desired result. $w^{\prime} \equiv 0$ implies $w \equiv$ const., which may be excluded. Hence we may assume that $N\left(r, 0, w^{\prime}\right)$ is of order at most $\rho$. Hence $T\left(r, w^{\prime}\right)$, that is, $w^{\prime}$ is of order at most $\rho$.

In our final step we can make use of Clunie's lemma in its original form and then of Lemma 1 . Then we have the desired result.

§4. Remarks. For $w^{\prime n}=a_{2} w^{2}+a_{1} w+a_{0}(n \geqq 3)$ or $w^{\prime n}=a_{1} w+a_{0}(n \geqq 2)$ we can prove the corresponding facts quite easily by the same method. However in the former case we can make use of Toda's results [5].

In the general case

$$
w^{\prime n}=\sum_{j=0}^{n-k} a_{j} w^{\jmath}, \quad 1 \leqq k \leqq n-1
$$

our method needs very laborious works.

If $T\left(r, a_{j}\right)=O(\log r)^{2}$ for $j=0,1,2,3$, then our theorem holds without exception. This is mainly due to Hayman's result in [3], although we need a further investigation for algebroid cases.

\section{REFERENCES}

[1] J. Clunie, On integral and meromorphic functions, J. London Math. Soc. 37 (1962), 17-27.

[2] F. Gackstatter and I. Laine, Zur Theorie der gewöhnlichen Differentialgleichungen im Komplexen, Ann. Pol. Math. 38 (1980), 259-287.

[3] W.K. HAYMAN, On the characteristic of functions meromorphic in the plane and of their integrals, Proc. London Math. Soc. 14A (1965), 93-128.

[4] W.K. Hayman, Research problems in function theory, Athlone Press, 1967.

[5] N. TodA, On the functional equation $\sum_{\imath=0}^{p} a_{\imath} f_{i} n_{\imath}=1$, Tôhoku Math. J. 23 (1971), 289-299.

[6] G. Valiron, Sur la dérivée des fonctions algebroides, Bull. Soc. Math. France 59 (1931), 17-39.

Department of Mathematics

TOKYO INSTITUTE OF TECHNOLOGY

Oh-OKayama, Meguro-ku, Tokyo, JAPAN 